\author{
Jen-San Chen \\ Professor, \\ Department of Mechanical Engineering, \\ National Taiwan University, \\ Taipei, Taiwan 10617
}

\section{On the Internal Resonance of a Spinning Disk Under Space-Fixed Pulsating Edge Loads}

Internal resonance between a pair of forward and backward modes of a spinning disk under space-fixed pulsating edge loads is investigated by means of multiple scale method. It is found that internal resonance can occur only at certain rotation speeds at which the natural frequency of the forward mode is close to three times the natural frequency of the backward mode and the excitation frequency is close to twice the frequency of the backward mode. For a light damping case the trivial solution can lose stability via both pitchfork as well as Hopf bifurcations when frequency detuning of the edge load is varied. On the other hand, nontrivial solutions experience both saddle-node and Hopf bifurcations. When the damping is increased, the Hopf bifurcations along the trivial solution path disappear. Furthermore, there exists a certain value of damping beyond which no nontrivial solution is possible. Single-mode resonance is also briefly discussed for comparison. [DOI: 10.1115/1.1408616]

\section{Introduction}

The vibration analysis of a spinning disk under space-fixed edge loads attracts attention because of its possible application in such fields as circular saw cutting and grind wheel operation. Carlin and his co-workers' investigation [1] appears to be the first paper attempting to calculate the natural frequencies of a spinning disk under a concentrated radial edge load. Radcliffe and Mote [2] extended the work of [1] by considering a general concentrated edge load with both radial and tangential components. Chen $[3,4]$ reformulated the problem with emphasis on the effects of relative motion between the disk and the edge load on the stability and natural frequencies of the loaded disk. Recently Chen [5] extended these analyses by considering the parametric resonance of a spinning disk under space-fixed pulsating edge loads.

The plate model employed in [5] ignored the effect of membrane stretching. As a consequence the equation of motion is linear in terms of the transverse deflection and the stiffness term involves a periodic coefficient which is due to the pulsating edge load. This linearized model imposes two limits on the applicability of the parametric resonance theory presented in [5]. First of all, while the linearized model can predict the onset of parametric resonance, it cannot predict the amplitude of steady-state vibration after parametric resonance occurs. Secondly, it cannot account for the complicated internal resonance phenomenon which is due to interaction between modes coupled by the nonlinear effect.

In this paper we extend our previous work [5] to consider the nonlinear parametric resonance of a spinning disk under spacefixed pulsating edge loads. Membrane stretching effect is taken into account by employing von Karman's plate model. We focus our attention on the internal resonance between a pair of forward and backward traveling waves with the same number of nodal diameters and nodal circles. Galerkin's procedure is used to discretize the equations of motion. The multiple scale method is then used to study the steady-state behavior and the stability of the

Contributed by the Applied Mechanics Division of THE AMERICAN SOCIETY OF MECHANICAL ENGINEERS for publication in the ASME JOURNAL OF APPLIED MECHANICS. Manuscript received by the ASME Applied Mechanics Division, May 15 2000; final revision, May 31, 2001. Associate Editor: R. C. Benson. Discussion on the paper should be addressed to the Editor, Professor Lewis T. Wheeler, Department of Mechanical Engineering, University of Houston, Houston, TX 77204-4792, and will be accepted until four months after final publication of the paper itself in the ASME JOURNAL OF APPLIED MECHANICS. response. Both static and dynamic bifurcation phenomena are discussed. The effects of damping on the bifurcation points are also studied in detail.

\section{Equations of Motion}

We consider an elastic circular disk spinning with constant speed $\Omega$. The disk is "partially" clamped [6] at the inner radius $r=a$ and is subjected to a periodic radial load at the outer radius $r=b$. In circular saw and grinding wheel operations the radial load is applied on a small sector of the outer edge. We assume that this space-fixed edge load can be expanded in a Fourier series $\cos \gamma t \sum_{k=0}^{\infty} P_{k} \cos k \theta . P_{k}$ has the dimension of stress, and $\gamma$ is the excitation frequency of the in-plane edge load. The direction of the edge load remains unchanged when the disk vibrates laterally. The equations of motion of the spinning disk in terms of transverse displacement $w$ and stress function $\phi$ can be written as [7]

$$
\begin{gathered}
\rho h\left(w_{, t t}+2 \Omega w_{, t \theta}+\Omega^{2} w_{, \theta \theta}\right)+c_{f} w_{, t}+D \nabla^{4} w \\
=h\left\{w_{, r r}\left(r^{-1} \phi_{, r}+r^{-2} \phi_{, \theta \theta}\right)+\left(r^{-1} w_{, r}+r^{-2} w_{, \theta \theta}\right) \phi_{, r r}\right. \\
\left.-2\left(r^{-1} w_{, \theta}\right)_{, r}\left(r^{-1} \phi_{, \theta}\right)_{, r}-\rho \Omega^{2} r\left(\frac{r}{2} \nabla^{2} w+w_{, r}\right)\right\} \\
\nabla^{4} \phi=-E\left[w_{, r r}\left(r^{-1} w_{, r}+r^{-2} w_{, \theta \theta}\right)\right. \\
\left.+2 r^{-3} w_{, r \theta} w_{, \theta}-r^{-2}\left(w_{, r \theta}\right)^{2}-r^{-4}\left(w_{, \theta}\right)^{2}\right] \\
+2(1-\nu) \rho \Omega^{2}
\end{gathered}
$$

$(r, \theta)$ are space-fixed polar coordinates. The parameters $\rho, h, E, \nu$, and $D$ are the mass density, thickness, Young's modulus, Poisson ratio, and flexural rigidity of the disk, respectively. $c_{f}$ represents a space-fixed homogeneous damping due to the surrounding air. In writing Eq. (2) the in-plane inertia is neglected. These two equations are based on the famous von Karman's plate model, which accounts for the membrane stretching due to bending.

It is noted that the total deflection of the disk cannot be deemed as the sum of the deflection components due to each Fourier component in the series because the edge load affects the stiffness operator instead of the forcing term. However, in Chen's previous work [4,5] on linear response in a similar loading situation he 
observed through numerical simulation that the effects of each Fourier component on the stability properties of the spinning disk are additive. In other words, if a Fourier component causes unstable vibration, then so does the general loading containing this particular Fourier component. As a consequence, it is possible to predict the behavior of the loaded disk by considering the effect of the general edge load as the combined effects of its individual components. Along this line of thought we focus on the response of a spinning disk under a Fourier component $\cos \gamma t P_{k} \cos k \theta$ in the following discussion.

The partially clamped boundary conditions for transverse deflection $w$ at $r=a$ are

$$
\begin{gathered}
w=0 \\
w_{, r}=0 .
\end{gathered}
$$

The boundary conditions at $r=b$ are

$$
\begin{gathered}
\left(\nabla^{2} w\right)_{, r}+r^{-2}(1-\nu)\left(w_{, r \theta \theta}-r^{-1} w_{, \theta \theta}\right)+\frac{h P_{k}}{D} \cos \gamma t \cos k \theta w_{, r}=0 \\
w_{, r r}+\nu r^{-1}\left(w_{, r}+r^{-1} w_{, \theta \theta}\right)=0 .
\end{gathered}
$$

The in-plane boundary conditions at $r=b$ are

$$
\begin{gathered}
\sigma_{r \theta}=0, \\
\sigma_{r}=P_{k} \cos \gamma t \cos k \theta .
\end{gathered}
$$

We also require that

$$
\phi<\infty \text { at } r=0 .
$$

It is noted that while Eqs. (1) and (2) are nonlinear in terms of $w$, they are linear in $\phi$. Therefore we can divide the stress function $\phi$ in Eq. (2) into three parts:

$$
\phi=\phi_{1}+\phi_{2}+\phi_{3} .
$$

The first part $\phi_{1}$ accounts for the stretching effect due to the centrifugal force. The second part $\phi_{2}$ accounts for the edge load effect. The corresponding stress fields $\sigma_{r k}, \sigma_{\theta k}$, and $\sigma_{r \theta k}$ can be found in a standard elasticity monograph [8]. The third part $\phi_{3}$ involves nonlinear terms of $w$. After substituting Eq. (10) in Eqs. (1) and (2) and introducing the following dimensionless quantities,

$$
\begin{gathered}
t^{*}=\frac{t}{b^{2}} \sqrt{\frac{D}{\rho h}}, \quad \Omega^{*}=\Omega b^{2} \sqrt{\frac{\rho h}{D}} \\
\gamma^{*}=\gamma b^{2} \sqrt{\frac{\rho h}{D}}, \quad r^{*}=\frac{r}{b}, \quad w^{*}=w \sqrt{\frac{b}{h^{3}}}, \\
\phi^{*}=\phi \frac{h}{D}, \quad c_{f}^{*}=c_{f} \frac{b^{3}}{24\left(1-\nu^{2}\right) \sqrt{\rho h^{3} D}}, \\
\varepsilon=12\left(1-\nu^{2}\right) \frac{h}{b}, \quad \eta=\frac{a}{b}, \\
\sigma_{r}^{*}=\frac{h b^{2}}{D} \sigma_{r}, \quad P_{k}^{*}=\frac{b^{3}}{12\left(1-\nu^{2}\right) D} P_{k},
\end{gathered}
$$

we can rewrite the equations of motion in the dimensionless form after dropping the asterisks for simplicity,

$$
\begin{aligned}
w_{, t t}+ & 2 \Omega w_{, t \theta}+\Omega^{2} w_{, \theta \theta}+\nabla^{4} w+L w+2 \varepsilon c_{f} w_{, t}+\varepsilon P_{k} \cos \gamma t L_{k} w \\
= & w_{, r r}\left(r^{-1} \phi_{3, r}+r^{-2} \phi_{3, \theta \theta}\right)+\left(r^{-1} w_{, r}+r^{-2} w_{, \theta \theta}\right) \phi_{3, r r} \\
& -2\left(r^{-1} w_{, \theta}\right)_{, r}\left(r^{-1} \phi_{3, \theta}\right)_{, r} \\
\nabla^{4} \phi_{3}= & -\varepsilon\left[w_{, r r}\left(r^{-1} w_{, r}+r^{-2} w_{, \theta \theta}\right)\right. \\
& \left.+2 r^{-3} w_{, r \theta} w_{, \theta}-r^{-2}\left(w_{, r \theta}\right)^{2}-r^{-4}\left(w_{, \theta}\right)^{2}\right] .
\end{aligned}
$$

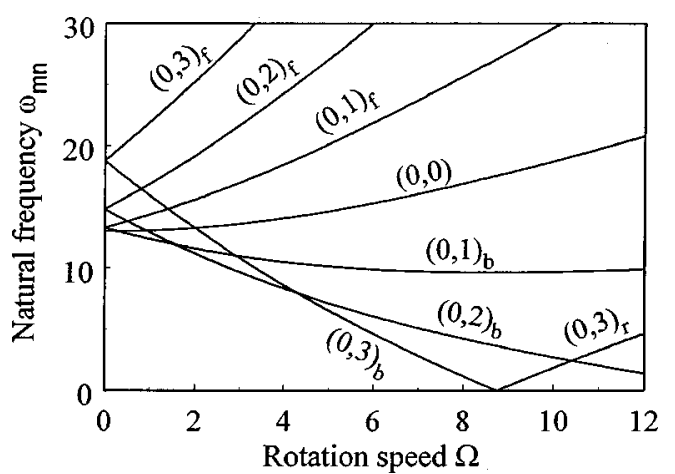

Fig. 1 Natural frequency loci of a freely spinning disk

$L_{k}$ is the membrane operator associated with the stress field due to the edge load, and $L$ is associated with the axisymmetrical stress field due to the centrifugal force,

$$
\begin{gathered}
L_{k} \equiv-\frac{1}{r}\left[\frac{\partial}{\partial r}\left(r \sigma_{r k} \frac{\partial}{\partial r}+\sigma_{r \theta k} \frac{\partial}{\partial \theta}\right)+\frac{\partial}{\partial \theta}\left(\sigma_{r \theta k} \frac{\partial}{\partial r}+\frac{1}{r} \sigma_{\theta k} \frac{\partial}{\partial \theta}\right)\right] \\
L \equiv-\frac{1}{r}\left[\frac{\partial}{\partial r}\left(r \sigma_{1 r} \frac{\partial}{\partial r}\right)+\frac{\partial}{\partial \theta}\left(\frac{1}{r} \sigma_{1 \theta} \frac{\partial}{\partial \theta}\right)\right]
\end{gathered}
$$

where

$$
\begin{gathered}
\sigma_{1 r}=\frac{3+\nu}{8} \Omega^{2}\left(1-r^{2}\right) \\
\sigma_{1 \theta}=\frac{1}{8} \Omega^{2}\left[(3+\nu)-(1+3 \nu) r^{2}\right] .
\end{gathered}
$$

When the nonlinear terms of $w$ are neglected in Eqs. (11) and (12), the equations reduce to the one considered in [5].

In the special case when $\varepsilon=0$, the solution $\phi_{3}$ in Eq. (12) is identically zero, and as a consequence Eq. (11) is reduced to

$$
w_{, t t}+2 \Omega w_{, t \theta}+\Omega^{2} w_{, \theta \theta}+\nabla^{4} w+L w=0 .
$$

Equation (17) is the equation of motion of a freely spinning disk. The natural frequency of a mode with $n$ nodal diameters and $m$ nodal circles is denoted by $\omega_{m n}$. The corresponding eigenfunction is complex and assumes the form

$$
w_{m n}(r, \theta)=R_{m n}(r) e^{i n \theta} .
$$

It is also noted that the eigenfunctions $w_{m n}$ are orthonormal.

Figure 1 shows the natural frequency loci of a spinning disk as the rotation speed varies. The clamping ratio $\eta$ is 0.5 , and the Poisson ratio $\nu$ is 0.27 . Only the modes with less than four nodal diameters are shown here. The subscripts $f, b$, and $r$ of the mode label $(m, n)$ represents forward, backward, and reflected modes, respectively [5].

\section{Discretization}

The linear analysis in [5] predicts that when the spinning disk is subject to a space-fixed distributed edge load $P_{2 n} \cos \gamma t \cos 2 n \theta$, single-mode parametric resonance can occur when the excitation frequency $\gamma$ is twice the natural frequency of an $(m, n)$ mode. Combination resonance involving two modes $(m, n)$ and $(p, q)$ can also occur when the numbers of nodal diameters $n$ and $q$ satisfy certain relations. In this paper we focus on the internal resonance between a pair of forward and backward $(m, n)$ modes excited by the in-plane edge load. We assume that solution $w(r, \theta, t)$ of Eqs. (11) and (12) can be approximated in terms of eigenfunctions $w_{m n}(r, \theta)$ as

$$
w(r, \theta, t)=c_{m n}(t) w_{m n}+\bar{c}_{m n}(t) \bar{w}_{m n} .
$$


Both $c_{m n}(t)$ and $w_{m n}(r, \theta)$ in Eq. (19) are complex functions, while the displacement $w(r, \theta, t)$ is a real function. $\bar{w}_{m n}$ represents the complex conjugate of $w_{m n}$. In order to solve $\phi_{3}$ in Eq. (12) we introduce a set of eigenfunctions $\phi_{m n}$ satisfying the following differential equation:

$$
\nabla^{4} \phi_{m n}-\beta_{m n}^{4} \phi_{m n}=0 .
$$

$\phi_{m n}$ satisfy the same boundary conditions as $\phi_{3}$ does. After expressing $\phi_{3}$ in terms of eigenfunction series $\phi_{m n}$ and following Galerkin's procedure, we can discretize Eqs. (11) and (12) into

$$
\begin{aligned}
\ddot{c}_{m n}+ & 2 i n \Omega \dot{c}_{m n}+\kappa_{m n} c_{m n}+2 \varepsilon c_{f} \dot{c}_{m n}+\varepsilon \mu \bar{c}_{m n} \cos \gamma t \\
& +\varepsilon \alpha\left|c_{m n}\right|^{2} c_{m n}=0
\end{aligned}
$$

where

$$
\begin{gathered}
\kappa_{m n}=\omega_{m n} \omega_{m \bar{n}} \\
\mu=\pi \int_{\eta}^{1}\left[r \sigma_{r(2 n)}\left(R_{m n, r}\right)^{2}+\left(\frac{n^{2} \sigma_{\theta(2 n)}}{r}-n \sigma_{r \theta(2 n), r}\right)\left(R_{m n}\right)^{2}\right] d r .
\end{gathered}
$$

$\omega_{m n}$ and $\omega_{m \bar{n}}$ are the natural frequencies of the backward and the forward modes, respectively. It is noted that $\omega_{m \bar{n}}$ is equal to $\omega_{m n}+2 n \Omega$. Constant $\alpha$ can be obtained via numerical integration involving eigenfunctions $w_{m n}$ and $\phi_{m n} .\left|c_{m n}\right|$ represents the absolute value of complex number $c_{m n}$.

\section{Multiple Scale Method}

We apply the method of multiple scale [9] to analyze the solution of Eq. (21). The method of multiple scale assumes an expansion of the solution in the form

$$
c_{m n}(t)=c_{m n}^{(0)}\left(t, T_{1}\right)+\varepsilon c_{m n}^{(1)}\left(t, T_{1}\right)+O\left(\varepsilon^{2}\right)
$$

where $T_{1} \equiv \epsilon t$. Substituting (24) into (21) and equating coefficients of like powers of $\epsilon$ yields

$$
\begin{gathered}
\varepsilon^{0}: \quad D_{0}^{2} c_{m n}^{(0)}+2 i n \Omega D_{0} c_{m n}^{(0)}+\kappa_{m n} c_{m n}^{(0)}=0 \\
\varepsilon^{1}: \quad D_{0}^{2} c_{m n}^{(1)}+2 i n \Omega D_{0} c_{m n}^{(1)}+\kappa_{m n} c_{m n}^{(1)} \\
=-2 D_{1} D_{0} c_{m n}^{(0)}-2 i n \Omega D_{1} c_{m n}^{(0)}-2 c_{f} D_{0} c_{m n}^{(0)} \\
-\alpha\left|c_{m n}^{(0)}\right|^{2} c_{m n}^{(0)}-\mu \bar{c}_{m n}^{(0)} \cos \gamma t
\end{gathered}
$$

where $D_{0} \equiv \partial / \partial t$, and $D_{1} \equiv \partial / \partial T_{1}$. The general solution of Eq. (25) can be written in the form

$$
c_{m n}^{(0)}=d_{1}\left(T_{1}\right) e^{i \omega_{m n} T_{0}}+d_{2}\left(T_{1}\right) e^{-i \omega_{m n} T_{0}} .
$$

Substituting (27) into the right-hand side of (26) we observe that there exist secular terms in three different cases. In the first case when $\omega_{m \bar{n}}$ is close to $3 \omega_{m n}$ and $\gamma$ is close to $2 \omega_{m n}$ internal resonance involving both modes will occur. In the second case when $\omega_{m \bar{n}}$ is close to $3 \omega_{m n}$ and $\gamma$ is close to $2 \omega_{m \bar{n}}$, only single-mode resonance will be induced. In the third case when $\omega_{m \bar{n}}$ is away from $3 \omega_{m n}$ and $\gamma$ is close to $2 \omega_{m n}$ or $2 \omega_{m \bar{n}}$, again only singlemode resonance is possible. The second and the third cases are the same in essence. No combination resonance of the sum or difference type is possible when only this pair of modes are considered [5]. In the following we focus on the internal resonance case.

\section{Internal Resonance: $\omega_{m \bar{n}}$ is close to $3 \omega_{m n}$ and $\gamma$ is close} to $2 \omega_{m n}$

In this case we assume that

$$
\begin{gathered}
\gamma=2 \omega_{m n}+\varepsilon \varsigma_{1} \\
\omega_{m \bar{n}}=3 \omega_{m n}+\varepsilon \varsigma_{2}
\end{gathered}
$$

where $\varsigma_{1}$ and $s_{2}$ are two independent detuning parameters. The secular terms of Eq. (26) can be eliminated if

$$
\begin{gathered}
2 i \hat{\omega}_{m n} D_{1} d_{1}+i 2 \omega_{m n} c_{f} d_{1}+\alpha d_{1}\left(\left|d_{1}\right|^{2}+2\left|d_{2}\right|^{2}\right) \\
-\frac{\mu}{2}\left(\bar{d}_{1} e^{\left.i \varsigma_{1} T_{1}+\bar{d}_{2} e^{i\left(\varsigma_{2}-\varsigma_{1}\right) T_{1}}\right)=0}\right. \\
2 i \hat{\omega}_{m n} D_{1} d_{2}+i 2 \omega_{m \bar{n}} c_{f} d_{2}-\alpha d_{2}\left(\left|d_{2}\right|^{2}+2\left|d_{1}\right|^{2}\right) \\
+\frac{\mu}{2} \bar{d}_{1} e^{i\left(\varsigma_{2}-\varsigma_{1}\right) T_{1}}=0
\end{gathered}
$$

where $\hat{\omega}_{m n}=1 / 2\left(\omega_{m n}+\omega_{m \bar{n}}\right)$. We express $d_{1}$ and $d_{2}$ in the forms

$$
\begin{aligned}
& d_{1}\left(T_{1}\right)=\frac{1}{2} a_{1}\left(T_{1}\right) e^{i \beta_{1}\left(T_{1}\right)} \\
& d_{2}\left(T_{1}\right)=\frac{1}{2} a_{2}\left(T_{1}\right) e^{i \beta_{2}\left(T_{1}\right)} .
\end{aligned}
$$

After substituting Eqs. (30) and (31) into Eqs. (28) and (29) we can conclude that the nontrivial steady-state solutions of $a_{1}, \beta_{1}$, $a_{2}$, and $\beta_{2}$ must satisfy the following equations:

$$
\begin{gathered}
4 \hat{\omega}_{m n} \varsigma_{1} a_{1}-\alpha a_{1}\left(a_{1}^{2}+2 a_{2}^{2}\right)+2 \mu\left(a_{1} \cos \psi_{1}+a_{2} \cos \psi_{2}\right)=0 \\
4 c_{f} \omega_{m n} a_{1}-\mu\left(a_{1} \sin \psi_{1}+a_{2} \sin \psi_{2}\right)=0 \\
4 \hat{\omega}_{m n}\left(2 \varsigma_{2}-3 \varsigma_{1}\right) a_{2}+\alpha a_{2}\left(a_{2}^{2}+2 a_{1}^{2}\right)-2 \mu a_{1} \cos \psi_{2}=0 \\
4 c_{f} \omega_{m \bar{n}} a_{2}+\mu a_{1} \sin \psi_{2}=0
\end{gathered}
$$

where

$$
\begin{gathered}
\psi_{1}=\varsigma_{1} T_{1}-2 \beta_{1} \\
\psi_{2}=\left(\varsigma_{2}-\varsigma_{1}\right) T_{1}-\beta_{1}-\beta_{2} .
\end{gathered}
$$

It is noted that single-mode resonance is not possible in this case. A straightforward solution procedure to solve Eqs. (32) to (35) for nontrivial solutions $a_{1}, a_{2}, \psi_{1}$, and $\psi_{2}$ is described briefly in the Appendix. The steady-state vibration of the spinning disk is then

$$
\begin{aligned}
w(r, \theta, t)= & R_{m n}(r)\left[a_{1} \cos \left(\frac{\gamma t}{2}-\frac{\psi_{1}}{2}+n \theta\right)\right. \\
& \left.+a_{2} \cos \left(\frac{3 \gamma t}{2}-\frac{\psi_{1}}{2}+\psi_{2}-n \theta\right)\right]+O(\varepsilon),
\end{aligned}
$$

which represents the superposition of two waves traveling in opposite directions. It is noted that the first term with $a_{1}$ in Eq. (38) is excited directly by the edge load, while the second term with $a_{2}$ is excited internally in a super-harmonic manner.

\section{Stability Analysis}

The stability of the steady-state solutions can be analyzed by expressing $d_{1}\left(T_{1}\right)$ and $d_{2}\left(T_{1}\right)$ as

$$
\begin{aligned}
& d_{1}\left(T_{1}\right)=d_{1}^{(s)}+\hat{d}_{1}\left(T_{1}\right) \\
& d_{2}\left(T_{1}\right)=d_{2}^{(s)}+\hat{d}_{2}\left(T_{1}\right) .
\end{aligned}
$$

$d_{1}^{(s)}$ and $d_{2}^{(s)}$ are the steady-state solutions. After substituting Eqs. (39) and (40) into Eqs. (28) and (29) and linearizing with respect to the variations $\hat{d}_{1}\left(T_{1}\right)$ and $\hat{d}_{2}\left(T_{1}\right)$ we can study the stability of the steady-state solutions. For instance, in order to study the stability of the trivial solutions we substitute

$$
\begin{gathered}
d_{1}\left(T_{1}\right)=\left[\hat{\alpha}_{1}\left(T_{1}\right)+i \hat{\beta}_{1}\left(T_{1}\right)\right] e^{\left(i \varsigma_{1} / 2\right) T_{1}} \\
d_{2}\left(T_{1}\right)=\left[\hat{\alpha}_{2}\left(T_{1}\right)+i \hat{\beta}_{2}\left(T_{1}\right)\right] e^{\left(-i 3 \varsigma_{1} / 2+i \varsigma_{2}\right) T_{1}}
\end{gathered}
$$

into Eqs. (28) and (29) to obtain the Jacobian matrix $[J]$ of the linearized equations as 


$$
[J]=\left[\begin{array}{cccc}
4 c_{f} \omega_{m n} & \mu-2 \varsigma_{1} \hat{\omega}_{m n} & 0 & \mu \\
\mu+2 \varsigma_{1} \hat{\omega}_{m n} & 4 c_{f} \omega_{m n} & \mu & 0 \\
0 & -\mu & 4 c_{f} \omega_{m \bar{n}} & \hat{\omega}_{m n}\left(6 \varsigma_{1}-4 \varsigma_{2}\right) \\
-\mu & 0 & -\hat{\omega}_{m n}\left(6 \varsigma_{1}-4 \varsigma_{2}\right) & 4 c_{f} \omega_{m \bar{n}}
\end{array}\right]
$$

By calculating the eigenvalues of the Jacobian matrix, we can determine the stability of the trivial solutions. The stability of the nontrivial solutions can be analyzed in a similar manner.

\section{Steady-State Solutions}

Figure 2 shows the amplitudes and phases of the steady-state solutions as functions of detuning parameter $s_{1}$ for the internal resonance between a pair of $(0,3)$ modes at $\Omega=3.3$. The parameters used in the calculation are $\varepsilon=0.01, \omega_{03}=9.9, \omega_{0 \overline{3}}=29.7$, $\mu=100, c_{f}=0.5$, and $\alpha=0.4$. The excitation frequency $\gamma=2 \omega_{03}$ $+\varepsilon \varsigma_{1}$. The solid and dashed curves represent stable and unstable solutions, respectively. The stable trivial solution undergoes a supercritical pitchfork bifurcation at $s_{1}=-3.11$ (point A). From point $\mathrm{A}$ the nontrivial solution branch is stable and undergoes a saddle-node bifurcation at point $\mathrm{H}\left(\mathrm{s}_{1}=0.90\right)$. On the other hand,
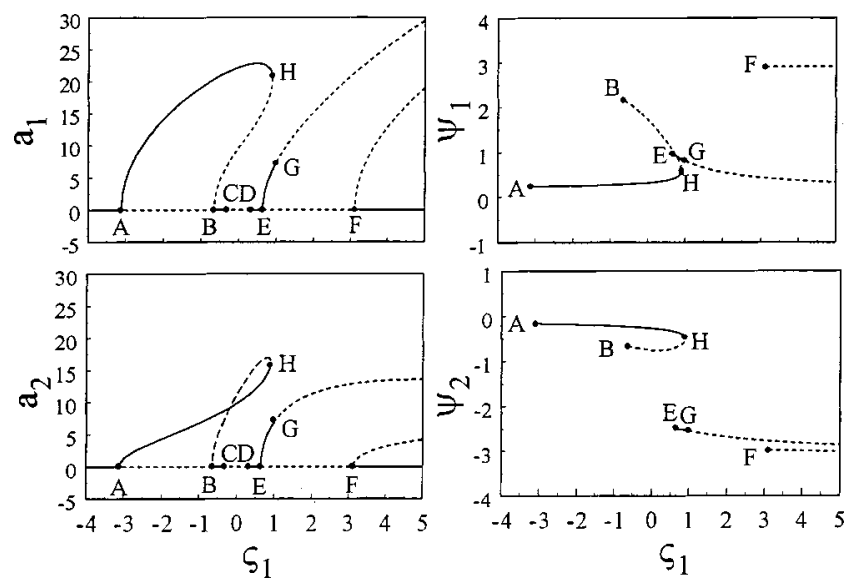

Fig. 2 Amplitudes and phases of the steady-state response. $\varepsilon=0.01, \Omega=3.3, \omega_{03}=9.9, \omega_{03}=29.7, \gamma=2 \omega_{03}+\varepsilon \varsigma_{1}, \mu=100, \alpha$ $=0.4$, and $c_{f}=0.5$.

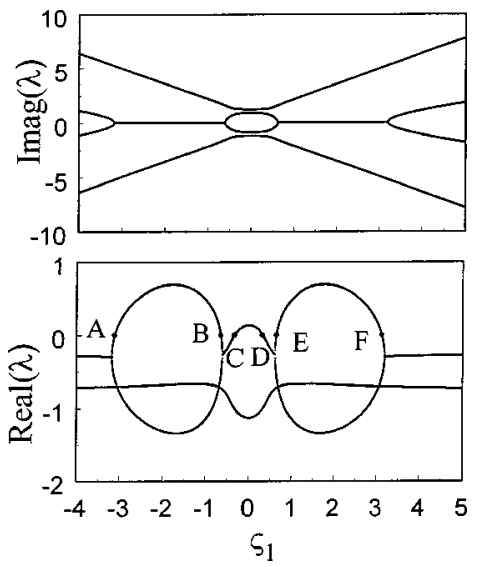

Fig. 3 Eigenvalues of the Jacobian matrix along the trivial solution path of Fig. 2 the unstable trivial solution from point A undergoes a subcritical pitchfork bifurcation at point $B\left(s_{1}=-0.64\right)$, creating an unstable nontrivial branch $\mathrm{BH}$ and a stable trivial branch BC. This stable trivial solution then loses stability via a supercritical Hopf bifurcation at $\varsigma_{1}=-0.33$ (point $\mathrm{C}$ ), creating a quasi-periodic solution $c_{03}$ which cannot be shown in Fig. 2. The unstable branch CD undergoes a supercritical Hopf bifurcation at point D $\left(s_{1}=0.33\right)$ and creates a stable trivial branch DE. The branch DE then undergoes a supercritical bifurcation at point E creating a stable nontrivial branch EG and unstable trivial branch EF. The stable branch EG then loses stability via a supercritical Hopf bifurcation at point $\mathrm{G}$ creating a stable quasi-periodic solution (which again cannot be shown in Fig. 2) and an unstable periodic solution. The trivial branch EF undergoes a subcritical pitchfork bifurcation at point $\mathrm{F}$ creating an unstable nontrivial branch and a stable trivial branch.

The bifurcation points along the trivial solution path can be verified by observing the eigenvalues $\lambda$ of the Jacobian matrix $[J]$ in Eq. (43) in Fig. 3. At points A, B, E, and F there exists a zero eigenvalue, which implies a pitchfork bifurcation. On the other hand at points $\mathrm{C}$ and $\mathrm{D}$ there exist a pair of purely imaginary eigenvalues and the real part loci of the eigenvalues cross the zero line "transversely," which implies a Hopf bifurcation [10].

To demonstrate the existence of quasiperiodic solutions predicted by the multiple scale analysis we use Runge-Kutta method to integrate Eq. (21) at $\varsigma_{1}=-0.32$ (a point slightly to the right of point $\mathrm{C}$ in Fig. 2) with initial conditions $c_{03}=0.1$ and $\dot{c}_{03}=0$. Figure $4(a)$ shows the response history of the real part of $c_{03}$ after a long period of time. Figure $4(b)$ shows its Poincare map recorded from $t=10,000$ to 12,000 with the sampling rate equal to the excitation frequency. The sampling points fill up a small strip

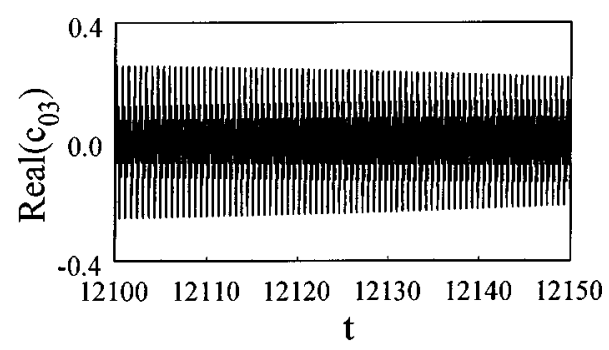

(a)

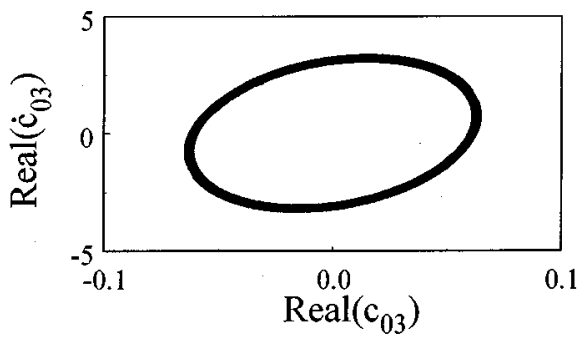

(b)

Fig. 4 (a) Quasi-periodic response and (b) the corresponding Poincare map for $s_{1}=-0.32$ with initial conditions $c_{03}=0.1$ and $\dot{c}_{03}=0$ 


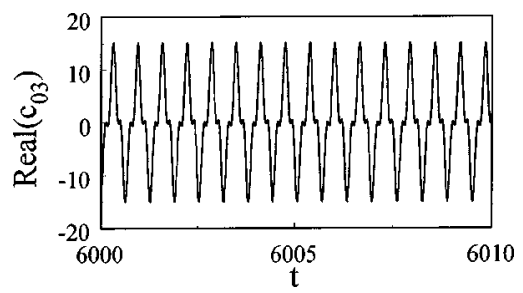

(a)

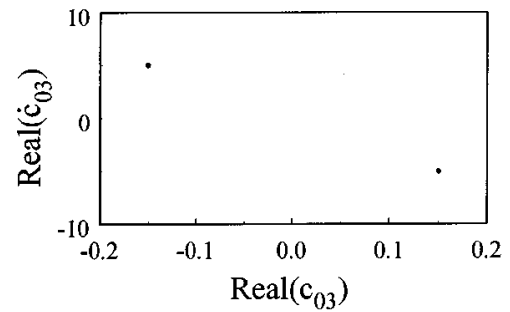

(b)

Fig. 5 (a) Periodic response and (b) the corresponding Poincare map for $\mathrm{s}_{1}=-0.32$ with initial conditions $c_{03}=20$ and $\dot{c}_{03}$ $=0$
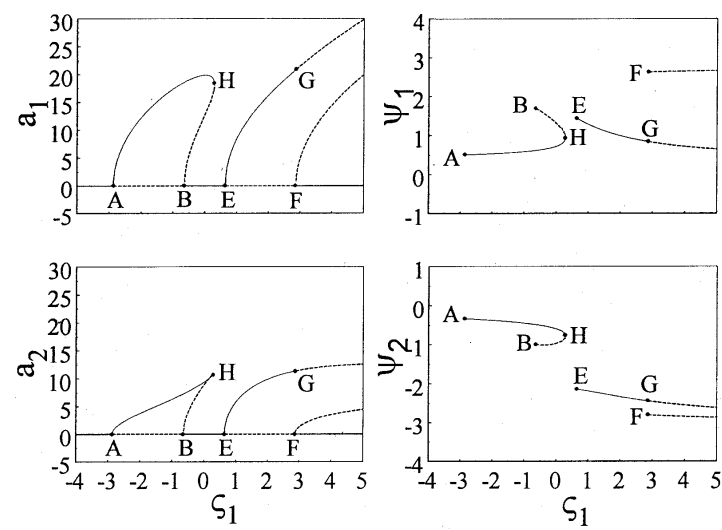

Fig. 6 Amplitudes and phases of the steady-state response. $\varepsilon=0.01, \Omega=3.3, \omega_{03}=9.9, \omega_{03}=29.7, \gamma=2 \omega_{03}+\varepsilon \varsigma_{1}, \mu=100, \alpha$ $=0.4$, and $c_{f}=1.0$.

around a closed curve, which implies the quasi-periodic feature of the response. If the initial condition of $c_{03}$ is changed from 0.1 to 20, the response then settles to the stable branch AH in Fig. 2, as shown in Fig. 5. The Poincare map in Fig. 5(b) records the sampling points from $t=4000$ to 6000 .

To observe the effects of damping we use the same parameters as those in Fig. 2 but change the damping $c_{f}$ from 0.5 to 1 . The amplitudes and phases of the steady-state solutions are shown in Fig. 6. We observe that the two Hopf bifurcation points $C$ and $D$ approach each other until they coalesce and disappear. Furthermore the Hopf bifurcation point $\mathrm{G}$ on the nontrivial solution branch moves to the right resulting in a longer stable branch EG.

\section{Bifurcation Points}

The observations on the eigenvalue loci in Fig. 3 allow us to predict the bifurcation points on the trivial solution path analytically. For the Hopf bifurcation to occur a pair of the eigenvalues of the Jacobian matrix (43) must be purely imaginary. By RouthHurwitz criterion we conclude that the detuning parameter $\varsigma_{1 H}$ must satisfy the equation

$$
A_{2 H} \varsigma_{1 H}^{4}+A_{1 H} \varsigma_{1 H}^{2}+A_{0 H}=0
$$

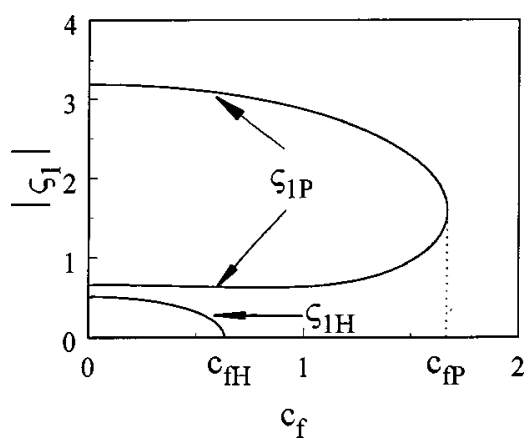

Fig. 7 Pitchfork and Hopf bifurcation points along the trivial solution path as functions of damping $c_{f}$

$$
\begin{gathered}
A_{2 H}=1024 \hat{\omega}_{m n}^{4} \omega_{m n} \omega_{m \bar{n}} \\
A_{1 H}=64 \hat{\omega}_{m n}^{2}\left[80 c_{f}^{2} \omega_{m n} \omega_{m \bar{n}} \hat{\omega}_{m n}^{2}+\mu\left(4 \hat{\omega}_{m n}^{2}+\omega_{m n} \omega_{m \bar{n}}\right)\right] \\
A_{0 H}=4096 c_{f}^{4} \omega_{m n} \omega_{m \bar{n}} \hat{\omega}_{m n}^{4}+64 c_{f}^{2} \mu^{2} \hat{\omega}_{m n}^{2}\left(\omega_{m n}^{2}+\omega_{m \bar{n}}^{2}\right) \\
-\mu^{4}\left(4 \hat{\omega}_{m n}^{2}-\omega_{m n} \omega_{m \bar{n}}\right) .
\end{gathered}
$$

The roots of Eq. (44) correspond to points C and D in Fig. 2. Similarly, the detuning parameter $s_{1 P}$ for pitchfork bifurcation must satisfy

$$
A_{2 P} \varsigma_{1 P}^{4}+A_{1 P} \varsigma_{1 P}^{2}+A_{0 P}=0
$$

where

$$
\begin{gathered}
A_{2 P}=144 \hat{\omega}_{m n}^{4} \\
A_{1 P}=4 \hat{\omega}_{m n}^{2}\left[16 c_{f}^{2}\left(8 \omega_{m n}^{2}+\omega_{m \bar{n}}^{2}\right)-15 \mu^{2}\right] \\
A_{0 P}=256 c_{f}^{4} \omega_{m n}^{2} \omega_{m \bar{n}}^{2}+16 c_{f}^{2} \mu^{2} \omega_{m \bar{n}}\left(2 \omega_{m n}-\omega_{m \bar{n}}\right)+\mu^{4} .
\end{gathered}
$$

The roots of Eq. (45) correspond to points A, B, E, and F in Fig. 2. In Fig. 7 we plot the absolute values of the roots of Eqs. (44) and (45) as functions of damping $c_{f}$. It is observed that Hopf bifurcation ceases to exist as $c_{f}$ exceeds 0.63 , while pitchfork bifurcation ceases to exist as $c_{f}$ exceeds 1.67 . In other words, no nontrivial solution is possible when $c_{f}$ is greater than 1.67. These two special dampings, denoted by $c_{f H}$ and $c_{f P}$, respectively, are proportional to $\mu$, and can be obtained by solving

$$
\begin{gathered}
A_{0 H}=0 \\
A_{1 P}^{2}-4 A_{2 P} A_{0 P}=0,
\end{gathered}
$$

respectively. Both Eqs. (46) and (47) are quadratic equations of $c_{f}^{2}$.

Single Mode Resonance: $\omega_{m \bar{n}}$ is close to $3 \omega_{m n}$ and $\gamma$ is close to $2 \omega_{m} \bar{n}$

In this case we assume that

$$
\gamma=2 \omega_{m \bar{n}}+\varepsilon \varsigma_{1} .
$$

The secular terms of Eq. (26) can be eliminated if

$$
2 i \hat{\omega}_{m n} D_{1} d_{1}+i 2 \omega_{m n} c_{f} d_{1}+\alpha d_{1}\left(\left|d_{1}\right|^{2}+2\left|d_{2}\right|^{2}\right)=0
$$

$2 i \hat{\omega}_{m n} D_{1} d_{2}+i 2 \omega_{m \bar{n}} c_{f} d_{2}-\alpha d_{2}\left(\left|d_{1}\right|^{2}+2\left|d_{2}\right|^{2}\right)+\frac{\mu}{2} \bar{d}_{2} e^{-i \varsigma_{1} T_{1}}=0$.

Following a similar procedure as in the internal resonance case we can conclude that the steady-state solution of $a_{1}$ is zero and the nontrivial steady-state solution of $a_{2}$ and $\beta_{2}$ satisfy the following conditions:

$$
4 c_{f} \omega_{m \bar{n}}-\mu \sin \psi_{2}=0
$$




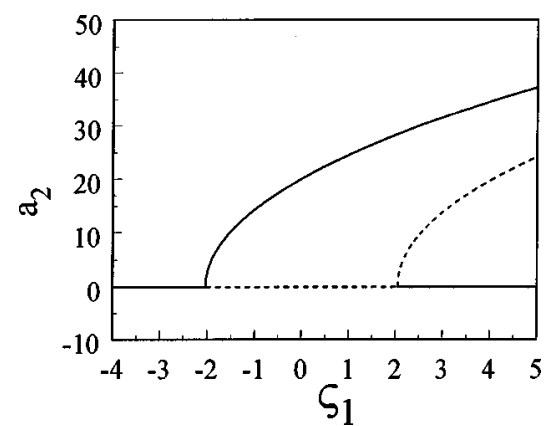

Fig. 8 Amplitude of the single-mode resonance. $\varepsilon=0.01, \Omega$ $=3.3, \omega_{03}=9.9, \omega_{03}=29.7, \gamma=2 \omega_{03}+\varepsilon \varsigma_{1}, \mu=100, \alpha=0.4$, and $c_{f}=0.5$.

$$
-\alpha a_{2}^{2}+2 \mu \cos \psi_{2}+4 \hat{\omega}_{m n} \varsigma_{1}=0
$$

where

$$
\psi_{2}=\mathrm{s}_{1} T_{1}+2 \beta_{2} .
$$

Therefore, only single-mode resonance is possible in this case. After eliminating $\psi_{2}$ from Eqs. (51) and (52), we derive the square of the steady-state amplitude $a_{2}$ as

$$
a_{2}^{2}=\frac{2}{\alpha}\left[2 \varsigma_{1} \hat{\omega}_{m n} \pm\left(\mu^{2}-16 c_{f}^{2} \omega_{m \bar{n}}^{2}\right)^{1 / 2}\right] .
$$

Nontrivial steady-state vibration begins to exist when

$$
\varsigma_{1}^{2} \leqslant \frac{\mu^{2}-16 c_{f}^{2} \omega_{m \bar{n}}^{2}}{4 \hat{\omega}_{m n}^{2}} .
$$

Equation (55) can also be predicted by the linear analysis in [5]. Figure 8 shows the amplitude $a_{2}$ of the steady-state vibration as a function of $s_{1}$ when the excitation frequency $\gamma$ is close to twice the natural frequency of mode $(0,3)_{f}$. The parameters used in the calculation are the same as those used in plotting Fig. 2.

\section{Conclusions}

In this paper we use multiple scale method to study the internal resonance between a pair of forward and backward modes of a spinning disk under space-fixed pulsating edge loads. Linear analysis in [5] predicts that only single-mode parametric resonance can occur when the excitation frequency is twice the natural frequency of the mode of interest. By considering the nonlinear effect resulting from von Karman's plate model, however, internal resonance is predicted in some special cases. Several conclusions are summarized as follows:

(a) Internal resonance can occur when the natural frequency of the forward mode is close to three times the natural frequency of the backward mode and the excitation frequency is close to twice the frequency of the backward mode. In the case when the exci- tation frequency is close to twice the frequency of the forward mode, on the other hand, only single-mode parametric resonance is possible.

(b) For light damping case the trivial solution can lose stability via both pitchfork as well as Hopf bifurcations when frequency detuning parameter is varied. On the other hand, nontrivial solutions experience both saddle-node and Hopf bifurcations.

(c) When the damping is increased the Hopf bifurcations along the trivial solution path disappear. There also exists a certain damping value beyond which no nontrivial solution is possible.

\section{Appendix}

By eliminating $\psi_{1}$ from Eqs. (34) and (35) we can derive an equation involving only $g=a_{1}^{2}$ and $f=a_{2}^{2}$ in the form

$$
p_{2}(f) g^{2}+p_{1}(f) g+p_{0}(f)=0 .
$$

With use of Eqs. (34) and (35), Eqs. (32) and (33) can be reduced to

$$
q_{4}(f) g^{4}+q_{3}(f) g^{3}+q_{2}(f) g^{2}+q_{1}(f) g+q_{0}(f)=0
$$

$p_{i}$ and $q_{i}$ are functions of $f$ only. Equations $(A 1)$ and $(A 2)$ can then be reduced to the following equation in terms of $f$ :

$$
\left(m_{2} p_{0}-m_{0} p_{2}\right)^{2}+\left(m_{2} p_{1}-m_{1} p_{2}\right)\left(m_{0} p_{1}-m_{1} p_{0}\right)=0
$$

where

$$
\begin{gathered}
m_{2}=p_{0}\left(p_{2} q_{3}-p_{1} q_{4}\right) \\
m_{1}=p_{0}\left(p_{2} q_{2}-p_{0} q_{4}\right)-p_{2}^{2} q_{0} \\
m_{0}=p_{0} p_{2} q_{1}-p_{1} p_{2} q_{0} .
\end{gathered}
$$

After solving $a_{2}$ from Eq. (A3), we can obtain $a_{1}$ from (A1).

\section{References}

[1] Carlin, J. F., Appl, F. C., Bridwell, H. C., and Dubois, R. P., 1975, "Effects of Tensioning on Buckling and Vibration of Circular Saw Blades," ASME J. Eng. Ind., 2, pp. 37-48.

[2] Radcliffe, C. J., and Mote, C. D., Jr., 1977, "Stability of Stationary and Rotating Discs Under Edge Load,” Int. J. Mech. Sci., 19, pp. 567-574.

[3] Chen, J.-S., 1994, "Stability Analysis of a Spinning Elastic Disk Under a Stationary Concentrated Edge Load," ASME J. Appl. Mech., 61, pp. 788-792.

[4] Chen, J.-S., 1996, "Vibration and Stability of a Spinning Disk Under Stationary Distributed Edge Loads," ASME J. Appl. Mech., 63, pp. 439-444.

[5] Chen, J.-S., 1997, "Parametric Resonance of a Spinning Disk Under SpaceFixed Pulsating Edge Loads," ASME J. Appl. Mech., 64, pp. 139-143.

[6] Benson, R. C., and Bogy, D. B., 1978, "Deflection of a Very Flexible Spinning Disk due to a Stationary Transverse Load," ASME J. Appl. Mech., 45, pp. 636-642.

[7] Nowinski, J. L., 1964, "Nonlinear Transverse Vibrations of a Spinning Disk," ASME J. Appl. Mech., 31, pp. 72-78.

[8] Coker, E. G., and Filon, L. N. G., 1957, A Treatise on Photo-Elasticity, Cambridge University Press, London.

[9] Nayfeh, A. H., and Mook, D. T., 1977, "Parametric Excitations of Linear Systems Having Many Degrees of Freedom," J. Acoust. Soc. Am., 62, pp. 375-381.

[10] Nayfeh, A. H., and Balachandran, B., 1994, Applied Nonlinear Dynamics, John Wiley and Sons, New York. 